\title{
Fabrication of anisotropic wetting surface with asymmetric structures using geometrical similarity and capillary force
}

\author{
Ye-Eun Lee ${ }^{1}$, Dong-Ki Lee ${ }^{2}$ and Young Hak Cho ${ }^{1,2^{*}}$ (D)
}

\begin{abstract}
In the present study, we proposed a fabrication process of anisotropic wetting surfaces with asymmetric grooved parallelogram structures employing basic MEMS processes and micro transfer molding process. A Si substrate and a PDMS mold from Si master were easily self-aligned due to geometrical similarity (all Si wafer have same crystal planes) so that parallelogram microchannels could be formed between the Si substrate and PDMS mold. The parallelogram channels were filled with SU-8 via capillary force, and then the SU-8 parallelogram structures were transferred to an adhesive polymer film. Finally, we obtained an anisotropic wetting surface with SU-8 parallelogram structures, which showed the characteristics of anisotropic wetting and asymmetric flow. The liquid droplets could easily wet in the longitudinal direction of the structures, and the asymmetric flow characteristics of the droplets in the direction perpendicular to the longitudinal direction of the structures could be observed.
\end{abstract}

Keywords: Anisotropic wetting surface, Asymmetric structure, Geometrical similarity, Capillary force

\section{Introduction}

Over the past few years, a large number of studies has been conducted on the properties of solid surfaces and the nature of liquids that repel solid surfaces depending upon the structure of the surface. Specifically, the anisotropic wetting characteristics are manifested by various directional and asymmetric surfaces present in nature. Several examples of directional textured surfaces with micro/nano-structures can be found in nature, such as butterflies' wings, water striders, and gecko's feet. The anisotropic wetting characteristics can be applied to various fields such as microfluidic devices, sensors, corrosion-resistant surfaces, and self-cleaning fields [111]. When anisotropic micro/nano- structures, such as micro-grooves or pillars, are fabricated on a surface, the asymmetric spreading and wetting behavior of a droplet can be observed. Some research groups fabricated groove structures or asymmetric column structures to

\footnotetext{
*Correspondence: yhcho@seoultech.ac.kr

${ }^{2}$ Institute of Precision Machinery Technology, Seoul National University

of Science and Technology, Seoul, South Korea

Full list of author information is available at the end of the article
}

realize an anisotropic wetting surface and characterized them intensively. For grooved structure, the liquid wetted the groove completely in the longitudinal direction [1-3], while the surface with asymmetric pillar structure showed anisotropic wetting characteristics and the liquid flowed well in the direction of the columnar structure [3-6]. Recently, there is a growing demand to understand the anisotropic wetting characteristics of such grooved structures and asymmetric structures, which is essential for studying and developing new devices and technologies employing which wetting, spreading, and adhesion can be controlled [7-11]. Therefore, fabrication technique of such surfaces with the characteristics of groove structures as well as asymmetric structures is helpful for the development of the above-mentioned new devices and technologies, especially in the fields of microfluidics and lab-on-a-chip.

In the current study, we proposed a novel method for the fabrication of an anisotropic wetting surface with asymmetric grooved structures (parallelogram structures) using basic MEMS processes (such as photolithography, anisotropic wet etching) and transfer molding. 
Through the evaluation of static and dynamic contact angles (CAs) of water droplet, it has been proved that the surface with parallelogram structures possesses the anisotropic wetting and asymmetric flow.

\section{Experimental}

\section{Geometric parameters of parallelogram structures}

Figure 1 presents the geometric parameters for anisotropic surface with parallelogram structures, which indicates the relationship between the width and interval of the pattern and shape of the parallelogram structures.

$$
\begin{aligned}
& W_{s}=W_{2}-W_{1} \\
& d=p+W_{1}
\end{aligned}
$$

where $W_{1}$ is the width of Si master, $W_{2}$ is the width of Si substrate, $p$ is the distance between pattern, $W_{s}$ is the width of parallelogram structure, and $d$ is the distance between the structures. $W_{s}$ is the difference of $W_{2}$ and $W_{1}$, as shown in Eq. (1). In Eq. (2), $p$ is an important factor which determines the distance between the structures $(d)$ of the anisotropic wetting surface and its size should be designed over $10 \mu \mathrm{m}$. If $p$ is too small $(<10 \mu \mathrm{m})$, it is difficult to achieve proper contact and bonding between the PDMS mold and Si substrate due to their insufficient contact area (Fig. 1). Furthermore, $h$ is the height of structure which was fixed to $25 \mu \mathrm{m}$, and $\theta$ is the angle between the structure and the film and was fixed to $54.7^{\circ}$, the angle between the (100) plane and the (111) plane of the silicon wafer.

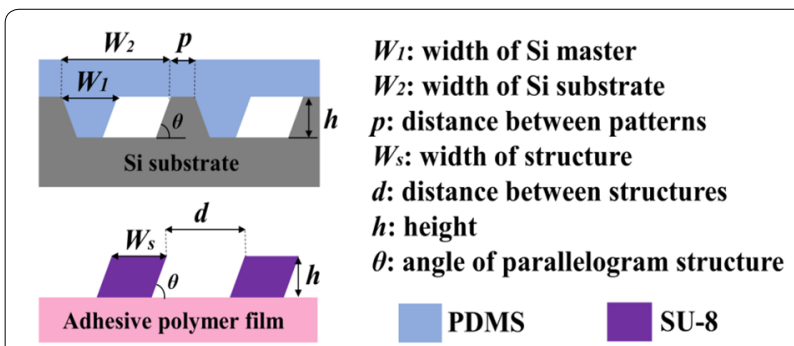

Fig. 1 Schematic view of anisotropic surface with parallelogram structures and their various geometrical parameters
In order to confirm the influence of the geometrical parameters of the parallelogram structure on the CA, two types of specimens were fabricated which had a parallelogram structure $\left(W_{s}\right)$ and widths $100 \mu \mathrm{m}$ and $200 \mu \mathrm{m}$, respectively. Also, to analyze the change in the wettability according to the distance of the parallelogram structure (d), each type of the specimen had 5 different values, viz. $100,150,200,250$, and $300 \mu \mathrm{m}$. Table 1 summarizes the values of the geometric parameters of the two types of specimens.

\section{Fabrication process}

In a previous study, we already reported the fabrication of microfluidic channels with various cross-sectional shapes, such as parallelogram, rhombus, pentagon and hexagon [12]. Also, the inverse-tapered structured oleophobic surface was formed using micromolding in capillaries and microtransfer molding [13]. By modifying these fabrication processes, in the present work, we propose a simple and novel fabrication method of anisotropic wetting surfaces with parallelogram structures.

Figure 2 shows the brief fabrication process of anisotropic wetting surface done using the basic MEMS processes, such as photolithography and anisotropic $\mathrm{KOH}$ wet etching, and the micro transfer molding process. The steps for fabrication of the anisotropic wetting surfaces with parallelogram structures are: (a) a thin film layer of $\mathrm{SiO}_{2}$ of thickness $5000 \AA$ was deposited on (100) single crystal Si wafer using thermal wet oxidation and patterned by photolithography and wet etching in buffered oxide etchant (BOE) solution; (b) the Si wafer was anisotropically etched with $\mathrm{KOH}$ solution at $80^{\circ} \mathrm{C}$; (c) the $\mathrm{SiO}_{2}$ layers from the $\mathrm{Si}$ master and $\mathrm{Si}$ substrate were removed by $\mathrm{BOE}$ solution, followed by formation of a silane (tridecafluoro-1,1,2,2tetrahydrooctyl trichlorosilane) coating on Si master; (d) a PDMS mold was made from the Si master; (e) the Si substrate and the PDMS mold were self-aligned and reversibly bonded using $\mathrm{O}_{2}$ plasma. A small amount of methanol (or DI water) was sprayed between the $\mathrm{Si}$ substrate and the PDMS mold to facilitate self-alignment. On evaporating the methanol on a hot-plate, microchannel arrays with parallelogram cross-section were formed whose interiors were

\begin{tabular}{|c|c|c|c|c|c|c|}
\hline \multirow[t]{2}{*}{ Structure type } & \multicolumn{6}{|c|}{ Geometrical parameters } \\
\hline & $\begin{array}{l}\text { Width of Si } \\
\text { master }\left(W_{1}\right) \\
{[\mu \mathrm{m}]}\end{array}$ & $\begin{array}{l}\text { Width of } \mathrm{Si} \\
\text { substrate }\left(W_{2}\right) \\
{[\mu \mathrm{m}]}\end{array}$ & $\begin{array}{l}\text { Width } \\
\text { of structure } \\
\left(W_{s}\right)[\mu \mathrm{m}]\end{array}$ & $\begin{array}{l}\text { Distance } \\
\text { between structures }(d)[\mu \mathrm{m}]\end{array}$ & Height $(h)[\mu \mathrm{m}]$ & $\begin{array}{l}\text { Angle of parallelogram } \\
\text { structure }(\theta)\left[^{\circ}\right]\end{array}$ \\
\hline Type 1 & 40 & 140 & 100 & $100,150,200,250,300$ & 25 & 54.7 \\
\hline Type 2 & 40 & 240 & 200 & & & \\
\hline
\end{tabular}

Table 1 Geometrical parameters of anisotropic surface with parallelogram structures 


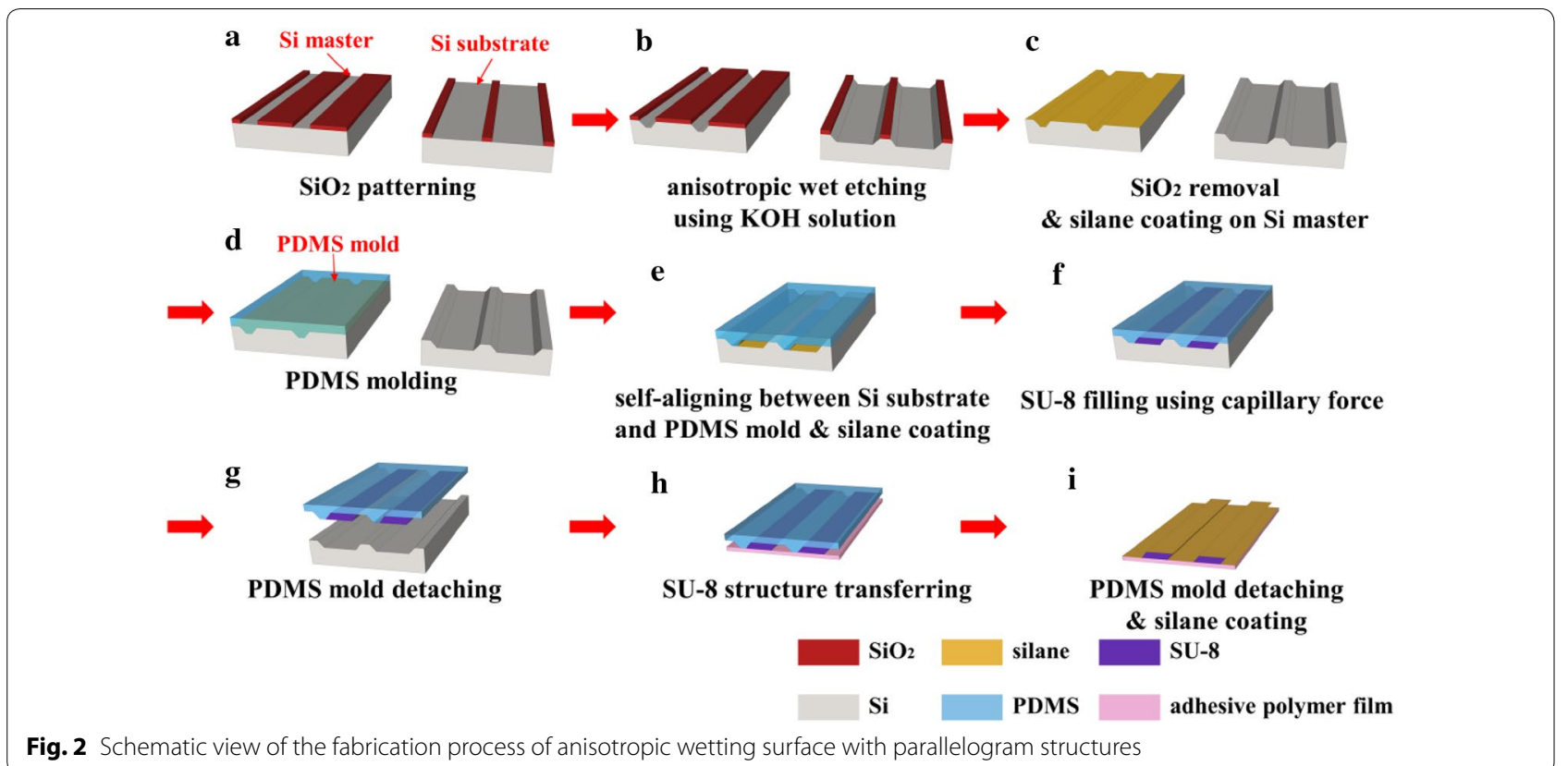

then coated with silane; (f) the microchannel arrays were then filled via capillary force with $\mathrm{SU}-8$, diluted with acetone, followed by hardening by baking and UV-exposure; (g) the SU-8 parallelogram structures, which were completely attached to PDMS mold, were peeled off from $\mathrm{Si}$ substrate; (h) when an adhesive polymer film was attached to the PDMS mold with the SU-8 structures, the SU-8 structures were transferred to the adhesive polymer film from the PDMS mold; (i) the adhesive polymer film with parallelogram structure was further coated with silane to reduce the surface energy.

\section{Contact angle measurement}

Wenzel and Cassie-Baxter suggested two different models based on theoretical method in order to explain phenomenon that the CA of drops on the fine structured surface is higher than on the flat surface $[14,15]$.

In the Wenzel state, the drop fills the voids below the liquid and thus occupies more surface area. The Wenzel CA, $\theta^{w}$, on the surface is given by

$$
\cos \theta^{w}=r \cos \theta_{0}
$$

where $\theta_{0}$ is an equilibrium CA on flat surface and $r$ is the roughness.

In the Cassie state, the drop rests upon the spaces with gas left in the voids below the drop. The surface area is less than it would be for a drop of the same volume and apparent CA on a flat surface or a rough surface in the Wenzel state. The Cassie $\mathrm{CA}, \theta^{\mathrm{c}}$, is given by

$$
\cos \theta^{c}=f\left(1+\cos \theta_{0}\right)-1
$$

where $f$ represents the fraction of the solid surface that is occupied by the ridges.

By measuring static and dynamic contact angles (CAs) of a water droplet on the surface with parallelogram structures using a CA goniometer (KSV CAM-200), the anisotropic wetting characteristics and the asymmetric flow characteristics of the droplet were quantitatively evaluated. A $\sim 10 \mu \mathrm{L}$ water droplet was dropped using a micropipette and the CAs were measured from the images captured by computer software. In these experiments, the static CAs were measured in the direction which is parallel to solid lines shown in Fig. 3a. To evaluate the asymmetric flow properties, the dynamic CAs [the advancing

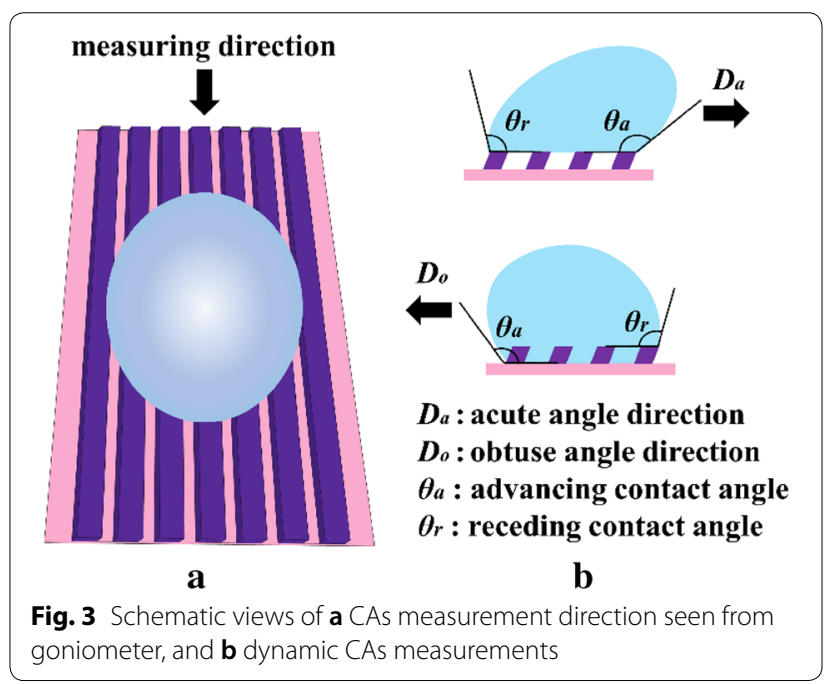


CAs $\left(\theta_{a}\right)$ and receding CAs $\left.\left(\theta_{r}\right)\right]$ and the contact angle hysteresis [CAH $\left(\theta_{a}-\theta_{r}\right)$ ] were measured by tilting the base unit at $1 \%$ s. The dynamic CAs were measured in both acute direction $\left(D_{a}\right)$ and obtuse direction $\left(D_{o}\right)$ of the surface due to asymmetry of the parallelogram structures. Figure $3 \mathrm{~b}$ shows a schematic view of the water droplet's shape when the anisotropic wetting surface was tilted in the acute direction and the obtuse direction.

\section{Results and discussion}

Figure 4 shows the scanning electron microscope (SEM) images of the self-aligned and bonded parallelogram microchannels (Fig. 4a-c) and the anisotropic wetting surfaces with parallelogram structures (Fig. 4d-f). Selfalignment between Si substrate and PDMS mold from $\mathrm{Si}$ master was possible because the $\mathrm{Si}$ substrate and $\mathrm{Si}$ master were fabricated from the same wafer, using anisotropic wet etching of $\mathrm{Si}$, and therefore, both the Si substrate and the master had the same structural angle and etching depth. The parallelogram microchannels were of $25 \mu \mathrm{m}$ height and $200 \mu \mathrm{m}$ width, whereas the distance between the channels were different, viz. 100, 150 and $200 \mu \mathrm{m}$. Figure $4 \mathrm{~d}-\mathrm{f}$ shows that the parallelogram structures with sharp edges were successfully transferred to the adhesive polymer films without any damage. The distance between the parallelogram structures $(d)$ was determined by the sum of the distance between the patterns $(p)$ and width of the Si master $\left(W_{1}\right)$.

To fill the SU-8 into the microchannel array, capillary force was used. The capillary pressure $P_{c}$ of the liquidair meniscus in such a rectangular microchannel can be expressed as [16]

$$
P_{c}=-\gamma\left(\frac{\cos \alpha_{b}+\cos \alpha_{t}}{h}+\frac{\cos \alpha_{l}+\cos \alpha_{r}}{w}\right)
$$

where $\gamma$ is the surface tension of the liquid, $\alpha_{b, t, l, r}$ are the contact angles of the liquid on the bottom, top, left and right wall, respectively, whereas $h$ and $w$ are the height $(25 \mu \mathrm{m})$ and width $(100$ and $200 \mu \mathrm{m})$ of the microchannel, respectively. The surface tension of SU-8 is $28.00 \mathrm{~mJ} /$ $\mathrm{m}^{2}$ at $25{ }^{\circ} \mathrm{C}$. The equilibrium CAs of SU-8 droplets on the silane-coated PDMS (top, left wall) and Si substrate (bottom, right wall) were $88.2^{\circ}$ and $78.1^{\circ}$. Finally, the calculated $P_{c}\left(-3.3\right.$ to $\left.-3.0 \times 10^{2} \mathrm{~N} / \mathrm{m}^{2}\right)$ has minus value, which means that SU-8 can go into microchannel without any external applied pressure.

To investigate the effect of the geometrical shape parameter $(d)$ and the structure width $\left(W_{s}\right)$ on the CA of water droplet, the CAs of the two types of specimens, classified according to the distance between the parallelogram structures (see Table 1) were measured. The mean values of the CAs measured at five arbitrary points on the same specimen were then calculated for further analysis. Figure 5 shows the static CAs for both the specimens.

As the width of the parallelogram structure increased from 100 to $200 \mu \mathrm{m}$, the static CA of Type 1 specimens were higher than that of Type 2 specimens regardless of the distance between the structures $(d)$. As the area of contact between the water droplet and the surface of the structure increased, the droplet wetted the solid surface with a high surface energy more easily, leading to a decrease in the static CA. In the Type 1 and Type 2 specimens presented in this research, the static CA decreased when the distance between the structures was over $250 \mu \mathrm{m}$ and $200 \mu \mathrm{m}$, respectively. Due to the geometrical shape characteristics of the parallelogram structures whose surface had two angles (acute angle and obtuse angle), the droplet tended to wet the bottom surface of the structures along the solid surface of the obtuse angle portion of the parallelogram structures. Therefore, the

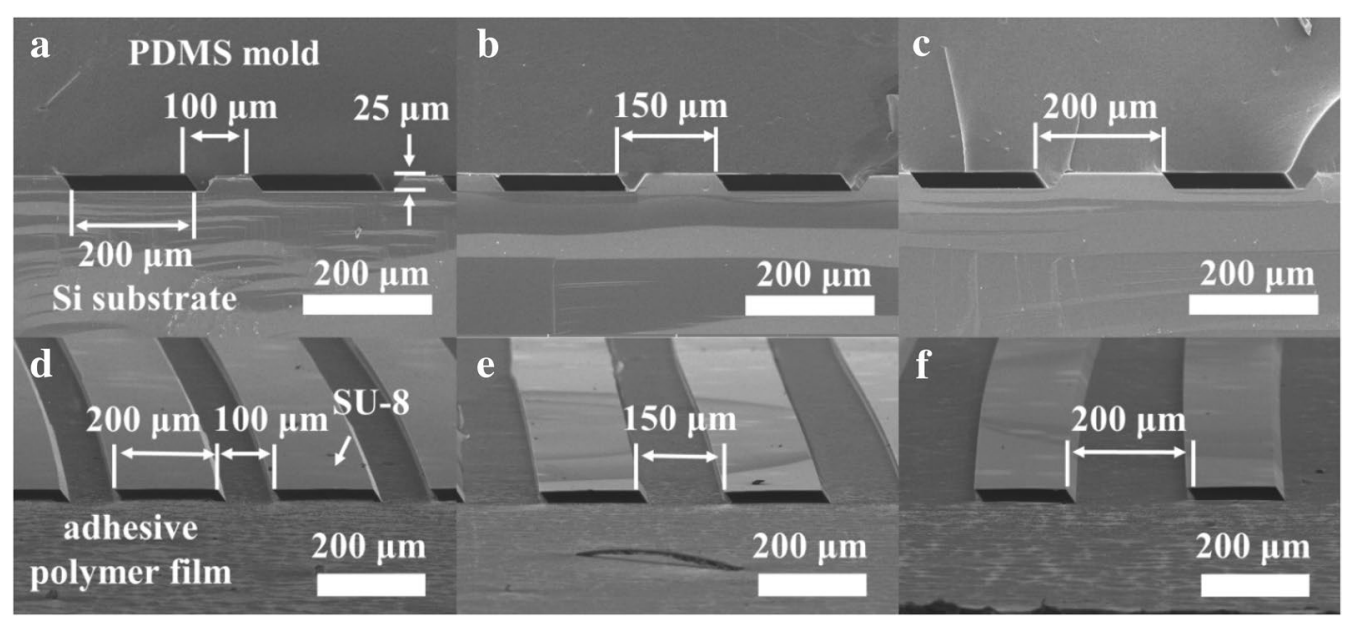

Fig. 4 SEM images of Type 2 specimen. a-c Aligned parallelogram channels, and $\mathbf{d}-\mathbf{f}$ parallelogram structures on the adhesive polymer film 


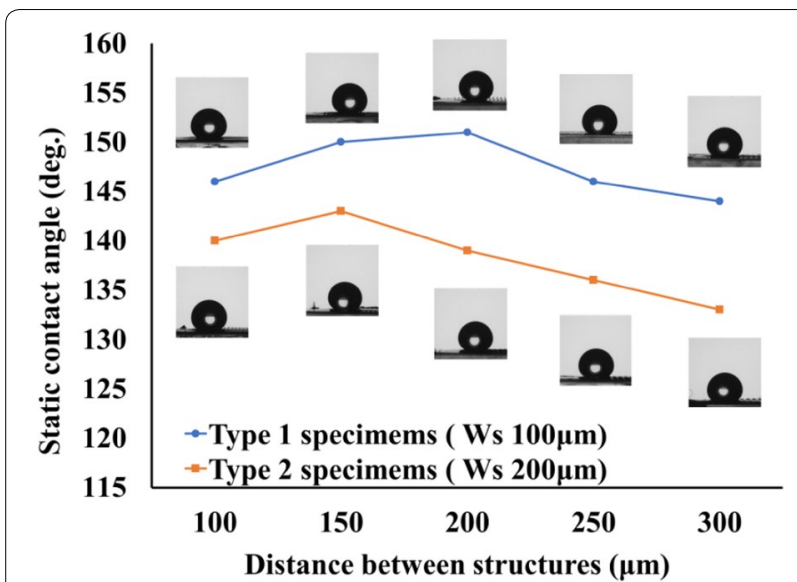

Fig. 5 A plot of static CAs of Type 1 and Type 2 specimens versus distance between the parallelogram structures

surface with parallelogram structures transformed easily from the Cassie-Baxter state to the Wenzel state, thereby causing the static CA to decrease. This result demonstrated that not only the width of structure and distance between the structures were important factors, but the geometrical shape of parallelogram structure was also significant in determining the wettability of the droplet.

In order to confirm the asymmetric flow characteristics on the surface with parallelogram structures, the dynamic CA of droplet was measured after tilting the specimens in both the acute angle $\left(D_{a}\right)$ and obtuse angle $\left(D_{o}\right)$ directions of the parallelogram. Figure 6 shows the contact angle hysteresis $(\mathrm{CAH})$ of the droplet when tilted to the direction of $D_{a}$ for both Type 1 and Type 2 specimens. Figure 6 shows the contact angle hysteresis $(\mathrm{CAH})$ of the droplet when tilted to the direction of $D_{a}$ for both Type 1 and Type 2 specimens, which is defined as the difference between

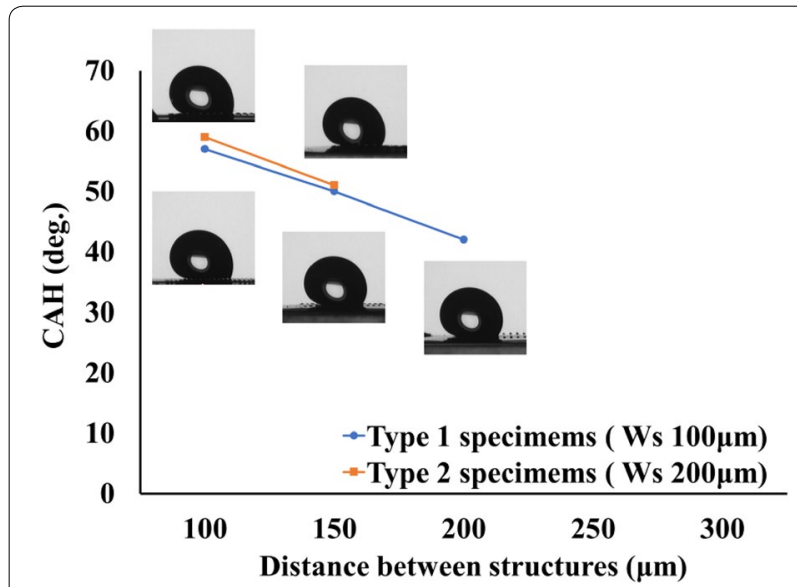

Fig. 6 A plot of contact angle hysteresis (CAH) for Type 1 and Type 2 specimens versus the distance between the parallelogram structures in the acute angle $\left(D_{a}\right)$ direction

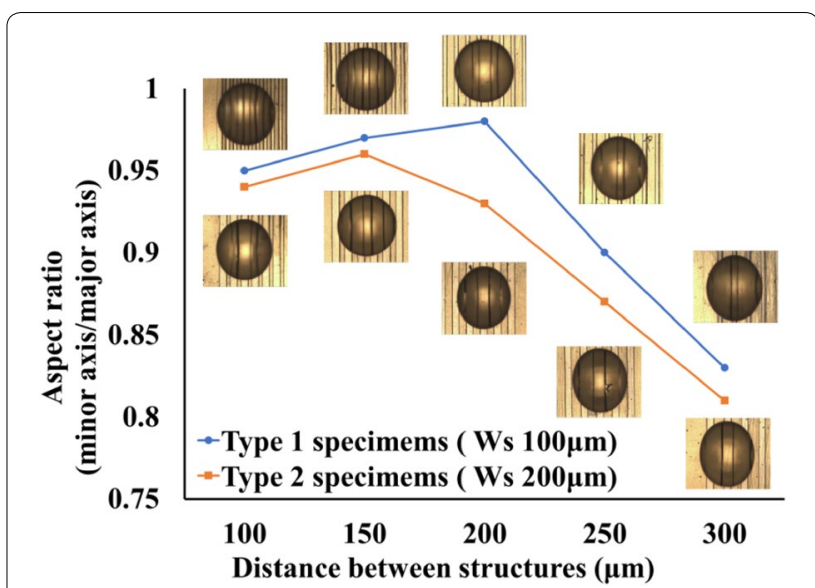

Fig. 7 Dependence of aspect ratio of water droplets on distance between the parallelogram structures

the advancing angle and the receding angle. The surface with static CA more than $90^{\circ}$ and small CAH is generally known to be hydrophobic. From Figs. 5 and 6, therefore, it can be said that the Type 1 specimen had highly hydrophobic surface in the direction of $D_{a}$. As the distance between the parallelogram structures increased, the $\mathrm{CAH}$ decreased. This is because the hydrophobicity of the anisotropic wetting surface became stronger to move to the acute angle $\left(D_{a}\right)$ direction of the parallelogram structure. For specimens where distance between the structures $(d)$ changed from Cassie-Baxter state to Wenzel state (e.g. when $d$ is $250,300 \mu \mathrm{m}$ for Type 1 specimens), although the tilting angle was $90^{\circ}$, the solid surface area in contact with the droplet widened; therefore, the droplet did not move and $\mathrm{CAH}$ could not be measured. Similarly, the water droplet also did not move and the $\mathrm{CAH}$ could not be measured for the Type 1 and Type 2 specimens when they were tilted up to $90^{\circ}$ in the obtuse angle $\left(D_{o}\right)$ direction. This was because the droplet could easily wet the bottom surface of the structure along the obtuse angle $\left(D_{o}\right)$ of the parallelogram structure due to gravity. This means that the water droplet was more likely to transit from the CassieBaxter state to the Wenzel state when the specimen was tilted in the obtuse angle $\left(D_{o}\right)$ direction.

From the measurement results of the dynamic CAs, it was proved that the surface with parallelogram structures showed the asymmetric flow characteristics because of the geometrical shape characteristics of the parallelogram [in the direction of acute angle $\left(D_{a}\right)$ and obtuse angle $\left.\left(D_{o}\right)\right]$.

The shape of water droplets on Type 1 and Type 2 specimens were also measured and analyzed to evaluate the anisotropic wetting characteristics of the surfaces with parallelogram structures. Figure 7 shows the top view shape of the water droplet on the anisotropic wetting 
surface and the aspect ratio of each droplet which is defined as the ratio of perpendicular direction (minor axis) to parallel direction (major axis) of parallelogram structures. The aspect ratio increased in both Type 1 and 2 specimens with increase in the width of the parallelogram structures, which indicated that the water droplet was close to the circle. This is because the solid area which was in contact with the water droplet in the Cassie-Baxter state decreased as the distance between the parallelogram structures increased. In other words, the liquid tended to maintain the circular shape due to the increased contact area between liquid and air.

On the other hand, as the distance between the parallelogram structures increased after transition to the Wenzel state, the aspect ratios for the Types 1 and 2 specimens decreased and the shape of the water droplet became elliptical. This is because the solid area in contact with the water droplet increased so the force by which the droplet wetted the solid surface in the longitudinal direction of the parallelogram structures became strong. This result showed that unlike the water droplet in the Cassie state, the water droplet in the Wenzel state displayed strong anisotropic wetting characteristics.

\section{Conclusions}

To conclude, we fabricated an anisotropic wetting surface with asymmetric grooved structures (parallelogram structures) using basic MEMS processes and transfer molding. To investigate the effect of the geometrical shape parameter $(d)$ and the structure width $\left(W_{s}\right)$ on the CA of water droplet, the CAs of two types of specimens were measured. The width of structure, the distance between the structures and the geometrical shape of parallelogram structure affected the wettability of the droplet. While measuring the dynamic CA of the droplet after tilting the specimens toward both the acute angle $\left(D_{a}\right)$ and the obtuse angle $\left(D_{o}\right)$ directions of the parallelogram, the surface showed different $\mathrm{CAH}$ value in the direction of the acute and obtuse angles, which signified that the asymmetric flow characteristics depended on the geometrical shape characteristics of the parallelogram. The shape of water droplets on Type 1 and Type 2 specimens were also measured and analyzed to evaluate the anisotropic wetting characteristics of the surfaces with parallelogram structures. In other words, through evaluation of the static and dynamic CAs and the aspect ratio of the water droplets, it can be confirmed that the surface with parallelogram structures had the anisotropic wetting and asymmetric flow. It is expected that the anisotropic wetting surface with asymmetric grooved structures can be applied to various fields such as microfluidic devices and self-cleaning where wetting, flowing, and adhesion can be controlled.

\section{Abbreviations}

$W_{1}$ : width of Si master; $W_{2}$ : width of Si substrate; $p$ : distance between patterns; $W_{s}:$ width of structure; $d$ : distance between structures; $h$ : height; $\theta$ : angle of parallelogram structure; $\theta^{w}$ : Wenzel contact angle; $\theta_{0}$ : equilibrium CA on flat surface; $r$ : roughness; $\theta^{c}$ : Cassie contact angle; $f$ : fraction of solid surface; $\theta_{a}$ : advancing contact angle; $\theta_{r}$ : receding contact angle; $\mathrm{CAH}\left(\theta_{a}-\theta_{r}\right)$ : contact angle hysteresis; $D_{a}$ : acute direction of surface; $D_{0}$ : obtuse direction of surface; $P_{c}$ : capillary pressure; $\gamma$ : surface tension of liquid; $a_{b}$ : contact angle of liquid on bottom wall; $a_{t}$ : contact angle of liquid on top wall; $a_{i}$ : contact angle of liquid on left wall; $a_{r}$ : contact angle of liquid on right wall; $w$ : width of microchannel.

\section{Acknowledgements}

This study was supported by the Research Program funded by the SeoulTech (Seoul National University of Science and Technology).

\section{Authors' contributions}

$Y L$ and $D L$ equally carried out the experiments and analyzed the experimental results, and YHC drafted the manuscript. All the authors discussed the proposed process and experimental results. All authors read and approved the final manuscript.

\section{Funding}

This study was supported by the Research Program funded by the SeoulTech (Seoul National University of Science and Technology).

Availability of data and materials

Not applicable.

Competing interests

The authors declare that they have no competing interests.

\section{Author details}

${ }^{1}$ Department of Mechanical System Design Engineering, Seoul National University of Science and Technology, Seoul, South Korea. ${ }^{2}$ Institute of Precision Machinery Technology, Seoul National University of Science and Technology, Seoul, South Korea.

Received: 7 Auqust 2019 Accepted: 10 October 2019

Published online: 15 October 2019

\section{References}

1. Chen Y, He B, Lee J, Patankar NA (2005) Anisotropy in the wetting of rough surfaces. J Colloid Interface Sci 281:458-464

2. Lee D, Lee J, Cho YH (2013) Effects of grooved surface with nano-ridges on amplification of hydrophobic property. Adv Mater Res 684:26-31

3. Xia D, Johnson LM, López GP (2012) Anisotropic wetting surfaces with one-dimensional and directional structures: fabrication approaches, wetting properties and potential applications. Adv Mater 24:1287-1302

4. Jokinen V, Leinikka M, Franssila S (2009) Microstructured surfaces for directional wetting. Adv Mater 21:4835-4838

5. Yang XM, Zhong ZW, Li EQ, Wang ZH, Xu W, Thoroddsen ST, Zhang XX (2013) Asymmetric liquid wetting and spreading on surfaces with slanted micro-pillar arrays. Soft Matter 9:11113-11119

6. Priest C, Albrecht TWJ, Sedev R, Ralston J (2009) Asymmetric wetting hysteresis on hydrophobic microstructured surfaces. Langmuir 25:5655-5660

7. Tawfick S, De Volder M, Copic D, Park SJ, Oliver CR, Polsen ES, Roberts MJ, Hart AJ (2012) Engineering of micro- and nanostructured surfaces with anisotropic geometries and properties. Adv Mater 24:1628-1674

8. Hancock MJ, Sekeroglu K, Demirel MC (2012) Bioinspired directional surfaces for adhesion, wetting, and transport. Adv Funct Mater 22:2223-2234

9. Kwak MK, Jeong H, Kim T, Yoon H, Suh KY (2010) Bio-inspired slanted polymer nanohairs for anisotropic wetting and directional dry adhesion. Soft Matter 6:1849-1857

10. Chu KH, Xiao R, Wang EN (2010) Uni-directional liquid spreading on asymmetric nanostructured surfaces. Nat Mater 9:413-417

11. Sun D, Böhringer KF (2019) Self-cleaning: from bio-inspired surface modification to MEMS/Microfluidics system integration. Micromachines 10:101 
12. Lee D, Kwon JY, Cho YH (2019) Fabrication of microfluidic channels with various cross-sectional shapes using anisotropic etching of Si and selfalignment. Appl Phys A 125:291

13. Hong KY, Lee D, Kim S, Cho YH (2019) Fabrication of anisotropically oleophobic surface with inverse-tapered structure using micromolding in capillaries and microtransfer molding. J Korean Soc Precis Eng 36(4):413-418

14. Wenzel RN (1936) Resistance of solid surfaces to wetting by water. Ind Eng Chem Res 28(8):988-994
15. Cassie ABD, Baxter S (1944) Wettability of porous surfaces. Trans Faraday Soc 40:546-551

16. Zimmermann M, Schmid H, Hunziker P, Delamarche E (2007) Capillary pumps for autonomous capillary systems. Lab Chip 7:119-125

\section{Publisher's Note}

Springer Nature remains neutral with regard to jurisdictional claims in published maps and institutional affiliations.

\section{Submit your manuscript to a SpringerOpen ${ }^{\circ}$ journal and benefit from:}

- Convenient online submission

- Rigorous peer review

- Open access: articles freely available online

- High visibility within the field

- Retaining the copyright to your article

Submit your next manuscript at $\boldsymbol{\nabla}$ springeropen.com 